\title{
Gravitational electric energy of a multilevel parking buildings in commercial and residential sectors proof of concept and prototype measurements
}

\begin{abstract}
Gravitation energy harvesting of climbed down objects is a promise to harvest unused potential energy from a host building or structure. Similarly, a dynamically dropper mechanism is proved to be a simple and effective electric energy generator, with many practical implementations in power, civil, and mechanical engineering. This research analyzes the prospect of using a mechanism tends to be near to the elevator structure, but without the counterweight for all possible energy harvesting. To achieve this aim, a dropper structure is supplemented with a new energy storage charging system for both the main kinetic and braking energy harvesting. A prototype of a scaled down system has been built and assumed that the original structure is a one level parking building and all the parked vehicles has a potential energy due to their climbed down to get out, which in turn is harvested by means of a DC generator. The primary goal is to maintain the control process for both; the speed and position by applying equivalent electric load to achieve maximum possible harvest energy from the host structure. Matching voltage and brake generative strategies is used to perform the energy harvesting concept. A polynomial modeling equation have been derived to form expressions of the attained power rate. The maximum power point (MPP) of the system has been extracted to highlight the optimization of system performance.
\end{abstract}

Keyword: Brake regenerative system; Brake regenerative in vehicles; Energy storage systems; Energy-efficient; Gravitational energy; Regenerative breaking energy utilization 